\section{Empowerment in the self-management of hypertension: challenges from the perspective of Iranian elderly patients}

Roghayeh Khezri ${ }^{1}$, Maryam Ravanipour ${ }^{2}$, Faezeh Jahanpour ${ }^{1}$, Maryam Barekat ${ }^{3}$, Somayeh Hosseiny ${ }^{4}$

\author{
Journal of Research \& Health \\ Social Development \& Health Promotion \\ Research Center \\ Vol. 7, No. 1, Jan \& Feb 2017 \\ Pages: 603- 613. \\ DOI: 10.18869/acadpub.jrh.7.1.603 \\ Original Article
}

1. Department of Nursing, School of Nursing and Midwifery, Bushehr University of Medical Sciences, Bushehr, Iran

2. Correspondence to: Department of Nursing, School of Nursing and Midwifery, The Persian Gulf Tropical Medicine Research Center, Bushehr University of Medical Sciences, Bushehr, Iran.

Email: ravanipour@bpums.ac.ir.

3. Department of Medicine, School of Medicine, Bushehr University of Medical Sciences, Bushehr, Iran

4. Department of Biostatistics School of Biostatistics, Bushehr University of Medical Sciences, Bushehr, Iran

Received: 30 Jan 2014

Accepted: 15 Jun 2014

How to cite this article: Khezri R, Ravanipour M, Jahanpour F, Barekat M, Hosseiny S. Empowerment in the self-management of hypertension: challenges from the perspective of Iranian elderly patients. $J$ Research \& Health2017; 7(1): 603- 613.

\begin{abstract}
Uncontrolled hypertension in the elderly causes various diseases and disability. This study was aimed to explore the challenges in self-management empowerment from the perspective of Iranian elderly patients with hypertension. Using exploratory descriptive approach, on 30 hypertension patients in Bushehr by purposive and convenience sampling method. First, qualitative data were collected through interviews with the elderly, and the quantitative data were then collected using a researcher-made questionnaire in three dimensions of knowledge, attitude and performance. In the qualitative phase, coding and summarizing of the data obtained from deep semi-structured interviews with the elderly led to five areas of awareness of changes, autonomy, satisfaction with performance, coping and sense of control. 7 men and 23 women participated in the qualitative phase, in which the average age of participants was $66.17 \%$ and $63 \%$ were married. Data analysis showed that the mean score of the elderly was equal to 10.17 and 58.26 for the dimensions of knowledge and performance respectively, which were at a weak level; and the mean attitude score was 9.96, which was at a moderate level. Given the challenges in empowering the management of hypertension in elderly, the planned training is recommended to be developed for the elderly and health care staff to achieve the main objective, i.e. the empowerment of the elderly through the self-management of hypertension.
\end{abstract}

Keywords: Elderly, Empowerment, Hypertension, Self-Management

\section{Introduction}

Hypertension is currently the health crisis in global health [1]. The prevalence of hypertension differs significantly around the world. It is equivalent to $11-30 \%$ in Latin America, $20-33 \%$ in Africa, 18$22 \%$ in USA, $44 \%$ in some European countries, and $25-30 \%$ in China, Korea and Taiwan [2]. In Iran, the frequency of hypertension is different in diverse cities so that the prevalence of systolic and diastolic hypertension has been reported to be equal to $69.5 \%$ and $54 \%$ in the population over 55 years of Sabzevar city, respectively [3]. The prevalence of hypertension was equal to $24.1 \%$ and $24.7 \%$ for men and women in Semnan province, respectively [4] and was $20.88 \%$ in the city of Gonabad [5]. According to the results of the MONICA project (monitoring trends and determinants in cardiovascular disease) in Bushehr, 97.7\% of the participants 
in this project have one of the cardiovascular risk factors, among which hypertension accounts for $24.5 \%$ [6]. Hypertension is strongly associated with increased rates of cardiovascular disease, is and chronic kidney disease (CKD) a major risk factor for stroke and is responsible for most deaths and illnesses, compared to other risk factors worldwide [7]. With increasing age, the gradual physiological changes take place in the cardiovascular system; for example vessel wall thickness increases and, as a result, vascular compliance decreases. The situation makes the elderly prone to hypertension [8]. On the other hand, the frequency of chronic diseases among the elderly and the subsequent increase in the cost of hospitalization, problems resulting from family separation and the risk of nosocomial infections today have led to home care. For these this reasons, the design of creative methods is necessary to achieve health outcomes by empowering the elderly patients in all aspects of care [9]. Recent studies have shown but hypertension can also be affected by the lifestyle and quality-of-life of individuals [10]. Hence, the education of healthy lifestyle to the elderly and their families has a particular importance. The motivation and cooperation of the elderly (especially participation in training sessions) are very important to achieve the objectives of education [11]. Several studies have highlighted educational needs and empowerment of people, including the need to educate them to cope with the disease and to perform self-care behaviors [12]. However, in some cases, nurses believe that patient education is at a moderate level (which is far from the optimal situation) [13], and that barriers to training the elderly patients include the lack of team cooperation, heavy workload, lack of staff time and lack of cooperation of the elderly in relation to education [14]. However, there are limited studies, especially those who examine the factors affecting the empowerment and challenges to empower the elderly in terms of chronic diseases, especially hypertension. Numerous studies have demonstrated the necessity to empower the elderly, in which individuals can be self empowered to manage their disease through education. A Norwegian study examined the process of empowering women with cancer, which uses four principles, i.e. raising awareness, learning objective knowledge, learning from the experience of others and discovering a new perspective in life. The results showed that self-care, with a focus on empowerment, can play a valuable contribution to the healing process of people with breast cancer [15]. Other studies on the empowerment show that it can improve HbA1c in diabetic people [16] and it can decrease blood pressure and improve the quality of life of people with hypertension [17]. These studies suggest somehow need to address the empowerment of patients with chronic diseases. On the other hand, for promotion of patients' quality of life and their perspective, using new educations and guidelines should be examined during clinical interventions [18]. In addition, the most important objectives of empowerment are to achieve self-competence and change one's view of self. Recognition of the person's competence increases the ability of elderly patients with chronic diseases to participate in solving the problem and makes significant changes in his/her well-being [19]. Also, it is known that other important objectives of psychological and personality empowerment are to increase critical knowledge, confidence, knowledge about the source of the problem and solutions, create the conditions for voluntary activities, and solve problems that affect the quality life [20]. Furthermore, studies show that the elderly patients, especially those with chronic disease, are unable to manage the disease, while the knowledge and awareness of personal and social resources help develop health and improve their quality of life. Therefore, it is essential for these patients to implement the empowerment intervention [9]. Because of existing few studies about improvement of elderly patients selfmanagement; Therefore, this study was designed to explore the challenges Iranian elderly patients with hypertension in selfmanagement empowerment from their perspective. 


\section{Method}

This study is an exploratory descriptive study (a kind of mixed method) aimed to examine the challenges related to self-management empowerment of elderly patients with hypertension in Bushehr city, the south of Iran, in managing their disease in 20122013. Exploratory method is used to identify perspectives, new insights and understandings, and explore factors associated with elderly patients with hypertension. Descriptive approach is used to describe information about a particular topic, and performs quantitative analysis of the data. Exploratory descriptive design is flexible because it gives opportunity to test all sides of the problem and attempts to establish new knowledge. This project does not seek to find causal relationships, but rather to study single variables and/or single populations $[21,22]$. On the other hand, exploratory descriptive studies are somehow belong to mixed methods, with regard to objectives and methodology, it was cleared this study was consistent with the sequential partial mixed method. This means that the study includes two qualitative and quantitative phases, which its quantitative phase is implemented sequentially with equal weight, after the implementation of the first qualitative phase $[23,24]$.

Accordingly, this study attempts to answer questions such as: "What problems the elderly patients with hypertension are faced on empowerment for self-management? And what conditions can contribute to the empowerment of elderly patients with hypertension, to improve their self-management?" It is important to note that an exploratory descriptive study is the starting point and seeks the generalization of the real world [21]. In this method, detailed descriptions of existing variables are collected, and then are used to justify and evaluate current conditions and activities [24].

We used this method too because in our study, we tried to answer questions such as: what are the challenges for the self-management empowerment of the elderly?

The study population consisted of the elderly patients with hypertension in the city of Bushehr.
Inclusion criteria for participants were: being aged 60 years or older, able to speak Persian, confirmed blood pressure $>90 / 140 \mathrm{~mm} \mathrm{Hg}$, or use of antihypertensive medication (by the patient) upon the prescription of a physician, and the desire to express his/her inner feelings towards the subject of study. The exclusion criteria were: being diagnosed with a mental illness or cognitive impairment and deafness, reluctance to interview or refusal to continue participation.

For the exploratory phase of the study, the directed content analysis approach was used to determine the challenges in self-management empowerment of elderly patients with hypertension, which was done on the basis of results of a study by Ravanipour on the concept of power and empowerment of elderly. This method was used in our study because we also wanted to examine and validate the concepts of self-management empowerment model, which included awareness of changes, coping, autonomy, role performance, sense of control, assumed satisfaction and self-management [25] In another new field of "Challenges in Empowerment of Elderly Patients with Hypertension from their Perspective", and ultimately contribute to the empowerment of the elderly to manage their disease $[19,26]$. In other words, the directed content analysis approach is used in cases that a theory or research done on the intended phenomenon, but in some aspects is deficient and needs to be more descriptive. Existing theory or research can help to focus research question. Bases of this theory are more structured than other types of content analysis, because researchers begin with basic concepts and key variables identified as initial codes of the issues [19]. Accordingly, we set semi-structured and openended questions to be directed, to achieve the goal based on the areas of self-management empowerment model.

Thus, the initial open-ended questions were first asked, such as: "Express your feelings about the day that a definitive diagnosis of your hypertension was made by the 
physician? Which difficulties you're faced in solving problems arising from the disease? And why? How have you solved these problems and challenges? With what things or what people?" Then, more targeted questions were asked on the basis of theoretical concepts of empowerment. For example, "How do you know your blood pressure goes up? (Awareness of changes); when your blood pressure goes up? What effect it can have on your everyday life? (Coping); who advice and help you to treat high blood pressure and why do you select him/her? (Autonomy); what do you do when your blood pressure go up? (Role performance); what do you do to better control your blood pressure? (Sense of control); in which case, will you be satisfied with your performance? (Assumed satisfaction); what programs and strategies did you design to better manage for your blood pressure? (Self-management)".

Therefore, sampling was made after getting a referral from the research deputy of university of medical sciences and offering it to the research environment. participants were selected based on a convenience and purposive sampling method and sampling continued for the qualitative phase until the categories were saturated and data were repeated. The participants were selected and collected in medical environments, including medical offices, clinics and coronary care unit $(\mathrm{CCU})$ units of hospitals. First, after we explained the objectives of the study and obtained written permission from the participants get involved in research and use audio recording during interviews, time and place for the interview was determined at the recommendation of the participants, and in the interview, after reintroducing and explaining the objectives of the study, interviews were made with elderly participants Based on the designed questions.

Based on Participants answer, for more clarify other questions asked of them. Next, immediately after the end of each interview, the recorded data were accurately listened in the shortest time and interviews were transcribed verbatim; and then, coding and data analysis were done by summarizing the codes in the more abstract categories using the content analysis approach, and finally, the challenges to selfmanagement empowerment were obtained from the perspective of the elderly in terms of issues. Credibility was enhanced through validation of emerging codes and categories in subsequent interviews, and debriefing with the research team. Using member checking, peer checking and maximum variation of sampling attested to the conformability of the findings. To enhance transferability, researchers discussed about the categories of data with some of the hypertension patients who did not participate in the study and confirmed the findings fit with the experiences [27,28].

Receiving data saturation, in this study, 15 individual interviews were made with nine of the participants until the collected data were been repetition of the previous data and new categories were not detected. It seemed that the sampling was terminated once the saturation was achieved, and then the interviews were analyzed.

After analyzing interview, according to the results of the interviews and the survey of the literature, a questionnaire was designed in the areas of the empowerment model [25,29], with the aim that "At what level is the challenge status of empowerment in the management of hypertension among the elderly in the areas of knowledge, attitude and performance?" It was scored on the Likert scale and was provided for new participants and was completed. Due to understanding only lesser-known areas, in exploratory descriptive studies, sample size is generally less importance, and usually at least a number of 30 participants is enough for the qualitative phase $[19,30]$. Data collection tool of the qualitative phase is a closedended questionnaire for self-management empowerment and demographic information. The questionnaire consists of three areas of knowledge, attitude and performance, and generally consists of 40 questions: 8 in the area of knowledge, 24 in the area of attitude, and 8 in the area of performance. In the sections of knowledge and performance, scoring was done as 1 and 2, which were 
assigned to the false or I-do-not-know cases and true ones, respectively. Thus, in the area of knowledge and performance, the scores were in the range of 8 to 16 . In the area of knowledge, if participants have earned a score of 8-10.65, 10.66-13.30 and 13.31-16, they were at weak, moderate and good levels, respectively. To obtain the levels, we first coded data at 3, 4 and 5 levels in SPSS, and then we divided the range of scores into three levels (weak, moderate and good) because the curve of data distribution at three levels is closer to the normal distribution [31]. The attitude section, which included fiveoption questions, was scored as Likert, from totally agree to totally disagree, with the scores of 1 to 5 . Consequently, the performance area was scored in the range of 24 to 120 . A person gaining a score of 24-55 will be at a weak level, a score of 56-88 at a moderate level, and a score of 89-120 at a good level. In this part, the levels are derived by dividing the difference in the range of scores into three. Dimensions of empowerment model Associated with the area of attitude, include seven areas of awareness of changes, autonomy, coping, role performance, assumed satisfaction, sense of control and selfmanagement. The validity of the questionnaire was measured by a survey from 10 specialist professors of nursing; and its reliability calculated by Cronbach's alpha was 0.89 .

The closed-ended questions of the questionnaire were completed by the elderly or, in case of inability to read, by the person accompanying him/her.

Thus, 30 questionnaires were completed, and data were analyzed using software SPSS-19 and the t-test and ANOVA descriptive statistics tests, in order to compare the mean scores of knowledge, attitude, and performance among different groups with different demographic variables (age groups, time of diagnosis and level of literacy). To respect the principles of research ethics, the purpose of the study was explained to the participants, and written informed consent was obtained for sampling. In addition, the voluntary nature of participation in the study and the confidentiality of the information were explained to the participants.

\section{Results}

For the qualitative phase of the study, nine patients were interviewed face to face. The mean age of the interviewees was 65 years; and 3 men and 6 women were presented at the interview. Most participants were married and lived with their spouses, and only the husbands of two women had died. Most of the participants were diagnosed between 5 and 10 years, and the majority of patients had a family history of hypertension.

Coding and summarizing the interviews led to the five areas of awareness of changes, autonomy, coping, satisfaction with performance, and sense of control.The elderly were partly awareness of the physical and mental effects of hypertension. Most of them highlighted the occurrence of headaches, dizziness, profuse sweating, and heaviness in the head, malaise, and sometimes nausea and vomiting. Although there were cases where they were Awareness of the changes due to hypertension in the body, they confused symptoms of hypertension with other symptoms. They, for example, believed that headache and dizziness were related to causes other than hypertension. Especially when it was asked: "In your opinion, what is blood pressure?" some said, " blood pressure is the blood fat. When the fat goes up, the individual suffers from high blood pressure". Those who had more information said different statements. For example, a 68-year-old says, "Every time my pressure goes up, I sweat, I am feeling dizzy, and I have a heavy feeling in my head." At the same time, many of the elderly were not awareness of the changes due to hypertension and its impact on the body's systems. All these statements indicate the high willingness of the elderly to learn and raise awareness about their disease.

Based on the discussions of the participants, it was seen that, regardless of their health status, the most important goal of most elderly were to obtain autonomy and meet their needs. The elderly, especially women, have a strong desire for independence, particularly in the management of their disease; and those with 
a sense of autonomy could feel autonomy, pride and vitality. They said, "If you're salaried, if you have a personal home, and if you're not in need of a burden to others, it is very satisfying ... but if you're in need, it seems that you're not allowed to make decisions for nothing! The elderly believed that one of the most important values is to maintain independence, which would lead to a sense of satisfaction.

It seemed that the elderly were making all their efforts so that they could be independent and could not depend on others to manage their disease. For this reason, they were planning to achieve this goal; and at the same time, they were seeking ways to solve problems and resolve dependencies.

Elders were trying to cope with the aging and the diseases associated with it. Many of the elderly participating in the study stated that they tell their secrets to God to cope with their hypertension and make peace. Some of them were trying to get more information so that they could have a comfortable life, despite illness, and could be less affected by complications of the disease. Although they did not like to taking medication and observe dietary, they had adopted somehow the current situation. A 60-year-old man says, "When you get a disease, you cope with it spontaneously, and there is no need to argue and talk." An elderly woman says, "Maybe you like eat the food without restrictions as in the past and like being not worried. that is the destiny of heaven. It may not always be the same. If you want not to get annoyed, you have to adapt to it."

In interviews, many of the elderly said that they are bound to take medications on time and adhere to low-salt and low-fat diet, which somehow was related to their attitude in a good performance as an elderly with hypertension, which was associated with satisfaction or dissatisfaction with his/her performance. On the other hand, some believe that hypertension affects their daily lives and is effective in the performance of their roles. An elderly woman says, "Since I got this disease, I go less the home of my children because my food should be low in salt and fat. I do not like that they become uncomfortable and prepare separate food for me. I do not like this, but I must admit my life." However, the respondents less discussed about satisfaction of drug use and their current conditions because they thought that talking about it is considered infidelity. if they were asked about their satisfaction with the current situation or condition, they immediately said that it was God's will that I've been sick, it is the divine will, and we have no objection ... or that they were not satisfied with their situation and were exhausted from taking the drug and observe food and pharmaceutical regular diet. In addition, when a question was asked about the satisfaction of the conditions, they mostly talked about the satisfaction of the conditions in the family, financial affairs and housing, and were less stated the satisfaction with their own and their performance. On the contrary, a 70-year-old woman however says," How can I not be upset when I have any home for myself? I have no money to go to the doctor. My children are in need to help themselves and don't have time to even meet me. You think, how can I be satisfied?" In fact, she considered satisfaction with performance in relation to the kind of satisfaction with life; and since she was totally unable to have a good performance in managing his life, inevitably, he has not well acted in managing the disease.

Many of the elderly felt that even though they are old, they themselves can control their disease and are able to gain control of your disease and most of the aging complications by complying with medication regimen, doing regular referral to a specialist and regular walking and having mobility. An elderly woman says, "You must be relaxed, observe the feeding behavior, and be active, I almost do these and I control my high blood pressure by eating medication on time." These people have a sense of inability and weakness due to aging, and the sense of empowerment increases if they have a job, the ability to meet their financial needs, the support of family, and the feeling of being valued. They were demanding respect from others. If they have 
high self-confidence and greater empowerment to manage their illness, they will try more and will have a greater sense of satisfaction and hope.

The participants in the quantitative phase were 30 patients aged 60 to 80 years. The mean age of the participants, who consisted of 7 men and 23 women, was 66.17.

In this study 63 percent were married and 20 percent were widowed. More than half of them (about 70\%) were illiterate, and few (about $30 \%$ ) are literate or are able to read and write. The diagnosis of hypertension was between $1-5$ years in more than $45 \%$, over 5 years in $55 \%$, and below one year in less than $3 \%$ of the participants.

Findings from the closed-ended questionnaire were analyzed in the dimensions of knowledge, attitude and performance, using descriptive statistics. Therelationshipbetweendemographic variables (mean age, sex, and diagnosis of the disease) and each of the dimensions of knowledge, attitude and performance were studied, which was not significant. Questions about the seven areas of empowerment model (awareness of changes, autonomy, coping, role performance, assumed satisfaction, sense of control, and self-management) were included in the dimension of attitude. The relationship between demographic variables and each of the dimensions were measured, which was not significant. The mean score on the dimension of knowledge indicate that the knowledge of the participants was at a weak level. In the attitude section, the mean score is 58.26, which expresses the mean attitude of participants toward education. In the dimension of performance, the mean score of 9.9 indicates poor performance of the participants in this area (Table 1).

Table 1 Mean, standard deviation and range of scores for knowledge, attitude and performance of subjects

\begin{tabular}{lccccc}
\hline Areas & $\begin{array}{c}\text { Number of } \\
\text { participants }\end{array}$ & Maximum & Minimum & Mean & Standard deviation \\
\hline Knowledge & 29 & 12.00 & 8.00 & 10.17 & 1.00 \\
Attitude & 26 & 77.00 & 42.00 & 58.26 & 7.84 \\
Performance & 30 & 9.00 & 13.00 & 9.96 & 1.06 \\
\hline
\end{tabular}

It was also stated that the areas of empowerment lied in the attitude section, and the score obtained from the following areas to change was as follows: the area of attitude at the mean level, the area of satisfaction and role performance at the mean level, coping at the good level, autonomy at the mean level and control at the weak level (Table 2).

Table 2 The range of scores and mean of the areas of empowerment model in the subjects

\begin{tabular}{lcccc}
\hline Areas & Number & Minimum & Maximum & Mean \\
\hline Awareness of changes & 28 & 3.00 & 10.00 & 5.78 \\
Role performance & 29 & 4.00 & 12.00 & 8.00 \\
Coping & 29 & 12.00 & 26.00 & 19.58 \\
Autonomy & 29 & 4.00 & 14.00 & 8.72 \\
Sense of control & 30 & 3.00 & 11.00 & 5.56 \\
Assumed satisfaction & 29 & 3.00 & 15.00 & 7.13 \\
Self-management & 30 & 2.00 & 7.00 & 3.76 \\
\hline
\end{tabular}

\section{Discussion}

In total, the results of the interviews revealed that challenges in self-management empowerment were primarily in the sense of lack of awareness about the disease and complications of hypertension control drugs, and feeling to need further information, and feeling of the effects of discomfort and stress on hypertension. As the quantitative findings clearly suggest, the information obtained from the questionnaires implies a lack of awareness. But despite all the problems, they had bound to the adherence to diet and medication; and to the extent that health insurance backed, they performed periodic checkups for blood tests. In case 
of disease control with medication and the compliance with low-fat and low-salt diet, they obtained the sense of their satisfaction with performance and the sense of having the ability to manage the disease; and in cases where they were unable to control their disease, they were unable to provide funding for treatment and had felt more loneliness, or had no certain job and income, then they develop a sense of inability and lack of self-satisfaction. The information obtained from the qualitative phase also indicates that the elderly patients are at the weak level of performance in the management of hypertension, but in the attitude section where empowerment dimensions were also included, it was seen that the elderly tend to achieve the empowerment to control the disease as well as the management of the disease; but to achieve this goal, they are involved in challenges, are trying to solve them, need support, and clearly express their needs in qualitative findings.

The findings of the qualitative phase express that the elderly were less awareness of the changes due to their disease and attributed most of the changes to aging and its effects as well as the complications of hypertension; and they stated that we need to learn more about their disease and the resulting changes so that, based on this knowledge, we have prompt and effective treatment. Some of them believed that headaches, dizziness, sweating are the common symptoms of hypertension, but there were those with vision problems, kidney or heart problems, who did not know that these cases are the complications of hypertension, and they mentioned lack of information about the disease and its complications and the need for more information and awareness for obtaining information. In this context, some questions were designed in the questionnaire, according to which there was even some elderly who did not know the meaning of hypertension or confused it with fat and artery clogging. The results of the qualitative phase confirmed the results of the qualitative phase. It can be said that the qualitative phase is the complement of the qualitative phase and somehow make sure the accuracy of the findings of the qualitative phase to discover the current challenges of the elderly. Finally, the findings of this quantitative study described the low and moderate levels of awareness. These results are consistent with the findings of the study by Hansen et al [32] They found that $79 \%$ of patients tend to acquire the necessary knowledge about disease progression, duration of disease and disease control practices. In a study performed in 2012 Heydari and Shahbazi [33] found that if the elderly have accurate information about proper practices of nutrition, exercise, sleep and rest, and proper use of drugs, many of their problems can be avoided or, if created, can be well-managed, and steps can be taken to improve their quality of life. The elderly with autonomy are able to gain better coping with their illness and will be in less trouble, thus coping with the existing conditions in order to take a role (role performance). The results of the qualitative phase showed that for the elderly, autonomy and independence are achieved by having housing, salaries and appropriate financial conditions. If they have good financial conditions, they follow the disease for control and treatment and try to achieve disease management and acquisition of knowledge. As the findings of the attitude dimension in the qualitative phase showed, the elderly have a positive approach to obtain management, but to achieve a good performance, which is the ability to manage hypertension, they need help and planning. In a study, Darvishpoor Kakhki also suggests that the elderly expect to maintain, in the elderly, the management style that has been formed for them in their lives, and be their own manager. The elderly want to take appropriate decisions through their intellectual faculties, and make them operational so that it can lead to a sense of identity and autonomy in the elderly [34]. In our study, the elderly expressed their satisfaction with the existing conditions when they are independent (especially in the financial and housing affairs) and have good treatment, family and mental condition. According to the statements of the elderly in the qualitative phase, it can be seen that 
having peace of mind, relationship with and support of their families, appropriate financial conditions and social protection and, in general satisfaction with life and existing conditions is very effective in the ability of individuals to manage disease, especially hypertension, in which stress and worry are the factors causing and intensifying hypertension. In his study, Borg also concluded that in the elderly who are less satisfied with their lives, their ability for disease self-management will be reduced thereafter [35]. In addition, life satisfaction has been associated with financial strength, family care, social support, performance independence and religion [36].

In general, findings from the qualitative phase of the questionnaire, includes knowledge, attitude and performance, were divided into three weak, moderate and good levels. The dimension of knowledge, which indicates the general information of the participants about the disease, was at a weak level. In the attitude section, the score means that attitude is at the moderate level, which represents an improvement in individuals' view toward awareness and treatment. This result is similar to the score of the attitude section (82.7 percent) in the study of Mazloumi et al [37] But in the dimension of performance, the obtained score indicated a low performance level of the participants. The attitude and knowledge of individuals may at moderate to high level; but to do them, they were faced with challenges for which they had not reached the desired satisfaction and sense of control over the disease and their overall situation, despite the completion of tasks. Individuals participating in the study of Mazloumi also have obtained the score of 57.72, which reflects the moderateto-low level of self-management performance in the patients .

There was no significant relationship between the score of knowledge, attitude and performance, and mean age, sex, and educational level. This result was somewhat close to the results of a study by Samadi et al [38] They found that there was no a strong correlation between the mean age and the scores of knowledge, attitude and performance. In addition, education level had no significant difference with the scores of performance and attitude. However it had a significant relationship with the score of their knowledge .

The score obtained in the qualitative phase was at the moderate level in the areas of awareness of changes, autonomy and satisfaction with performance, at a good level in the area of coping, and at a weak level in the area of sense of control. Findings indicated that the empowerment of individuals and autonomy, awareness of changes, sense of control, satisfaction with performance and power of self-management were at the moderate and low levels, and were at the good level just in the area of coping, which perhaps in a way reflects that individuals in this age are consistent with hypertension because of the experience gained from age and/or the assumption of chronic illness in the elderly.

The score of self-management was at the moderate level, suggesting self-management by the elderly to control high blood pressure is not desirable and also has no significant relationship with age, sex, education level and family history of the disease. The results indicate that the elderly needs more training for self-management. Mohammad Hassani et al also [33] found no significant relationship between power of self-management in patients with coronary artery disease and factors such as sex, age, employment, and income and education level.

Since our participants were selected using convenience and purposive sampling, the results cannot be generalized to the entire population of elderly patients with hypertension, although they gave us in-depth information about the challenges associated with their disease management. Accordingly, it is suggested that another study to be design based on self-management empowerment model, to determine the effect of this model on the management of hypertension in the elderly.

\section{Conclusion}

The results of this study suggest that elderly 
patients with hypertension are facing several challenges in the areas of awareness of changes, autonomy, coping, satisfaction with performance and sense of control, in order to manage their disease. Although they were made the best efforts to solve these problems, it seems that many situations were dominated in order to make independent or dependent a person. Having autonomy, adequate knowledge about the disease and how to control it, sense of control over life and disease, and life satisfaction, appropriate financial conditions, and the respect and attention of family and community to the elderly would be very effective in empowering the elderly and eventually achieving self-management in them; and the family and community should strive to achieve this goal.

\section{Acknowledgements}

We hereby acknowledge the generous cooperation of Mrs. Dr. Anaraki, head of Department of Family Health, the Deputy of Health in Bushehr Province, and the honorable Ms. Sarkhosh, health program expert for the elderly, the Health Deputy of Bushehr city, and all the honorable elderly who collaborated with us in this project.

\section{Contribution}

Study design: RKH, MR, FJ

Data collection and Data analysis: RKH, MR, $\mathrm{MB}, \mathrm{SH}$

Manuscript preparation:RKH, MR, FJ

\section{Conflict of Interest}

"The authors declare that they have no competing interests."

\section{Funding}

The author (s) received no financial support for the research, authorship and/or publication of this article.

\section{References}

1- WHO. A global brief on Hypertension, Silent killer, global public health crisis. 2013.

2- Haghdoost AA, Sadeghirad B, Rezazadeh Kermani M. Epidemiology and heterogeneity of hypertension in Iran: a systematic review. Archives of Iranian Medicine2008; 11(4): 444-52.

3- Pejman A, Najar L, Haidari A. The prevalence of hypertension in Sabzevar population. Journal of Sabzevar University of Medical Science2009; 15(1): 26.

4- Ghorbani R, Askandarian R, Malek M, RashidyPour A. Prevalence of hypertension among the adult population of Semnan province. Iranian Journal of Endocrinology \& Metabolism1387; 10(5): 495-503.

5- Naghavi M. Baraci shiue feshar khon dar shahr Gonabad. Journal Ofogh Danesh2001; 6(1): 66-77.

6- Amiri M, Emami S, Nabipour I, et al. Risk factors of cardiovascular diseases in Bushehr port on the basis of the WHO MONICA Project The persian Gulf healthy Heart Project. Iranian South Medical Journal2004; 6(2): 151-61.

7- National Heart Foundation of Australia ABN 98008419 761,3rd edition December 2010.Available at URL: http: // www.heartfoundation.org.au/SiteCollectionDocuments/ HypertensionGuidelines2008to2010Update.pdf. Accessed Sep 21, 2015.

8- Fakhrzadeh H, Sharifi F. Cardiovascular diseases in the elderly. Journal of Gorgan University of Medical Sciences2012; 14(3): 9-1.

9- Fotoukian Z, Mohammadi Shahboulaghi F, Fallahi Khoshknab M. Analytical on empowerment interventions in older people with chronic disease: A review literature. Journal Health Promotion Management2013; 2(4): 76-65.

10- Hamidizade S, Ahmadi F, Asghari M. Study effect of relaxation technique on anxiety and stress in elders with hypertension. Journal of Shahrekord Uuniversity of Medical Sciences2006; 8(2): 45-51.

11- World health organization. Dynamic aging. Mashhad: Ministry of health and medical education; 2005.

12- Stromberg A. The crucial role of patient education in heart failure. Eur J Heart Fail2005; 7(3): 363- 9.

13- Zamanzadeh V, Valizadeh L, Mohjal Aghdam A, Reza Gholi A.The patient education situational report: Nurses working in health centers-Tabriz. Journal of Tabriz Nursing and Midwifery2010; 5(19): 39-46.

14- Mazaheri E, Mohammadi R. Elderly patient education barriers from the perspective of health personnel of Ardabil university of medical sciences. Dena2006; 1(1): 75-82.

15- Stang I, Mittelmark MB. Learning as an empowerment process in breast cancer self-help groups. J Clin Nurs2009; 18(14): 2049-57.

16- Tang TS, Funnell MM, Brown MB, Kurlander JE. Self-management support in real-world settings: An empowerment-based intervention. Patient Educ Couns2010; 79(2): 178-84. 
17- Figar S, Galarza C, Petrlik E, et al. Effect of Education on blood pressure control in elderly persons: a randomized controlled trial. Am J Hypertens 2006; 19(7): 737-43.

18- Hatonen H. Patient education to support the selfmanagement of patients with mental illness. [thesis] Faculty of Medicine. Finland: University of Turku; 2010. 19- Hsieh HF, Shanon SE. Three approaches to qualitative content analysis. Qual Health Res2005; 15: 1277-88

20- Bakhshali Saatloo F, Taghdisi M, Shojaizade D, Baghaei R, Hosseini F. The effect of education based on empowerment model on knowledge, self-efficacy and practice of mothers with young girls for preventing of urinary tract infection. Journal of Research \& Health2014; 4(1): 584-91.

21- Mockford A. An exploratory descriptive study of the needs of parents after their young child is discharged from hospital following ad admission with an acute illness. [thesis] Wellington: Victoria University of Wellington; 2008.

22- Wood MJ, Ross KJC. Basic steps in planning nursing research. Boston: Jones \& Bartlett; 2006.

23- Leech NL, Onwuegbuzie AJ. A typology of mixed methods research designs. Quality \& Quantity2009; 43(2): 265-75

24- Mohammadpour A, Sadeghi R, Rezaie M. Mixed methods research as third methodological movement. Journal of Applied Sociology2010; 21 (2):77-100.

25- Ravanipour M, Salehi S, Taleghani F, Ali Abedi H, Schuurmans MJ, De Jong A. Sense of power among older people in Iran. Educational Gerontology2008; 34(10): 923-38.

26- Elo S, Kyngäs H. The qualitative content analysis process. $J$ Adv Nurs2008; 62(1): 107-15.

27- Speziale HS, Streubert HJ, Carpenter DR. Qualitative research in nursing: advansing the humanistic imperative. Wolters Kluwer. New York, London: Lippincott Williams \& Wilkins; 2011.

28- Holloway IW. Qualitative research for nurses. Australia: Blackwell science; 2002.

29- Ravanipour M, Salehi S, Taleghani F, Ali Abedi H. Power in old age: A qualitative study. Iranian Journal of Psychiatry and Clinical Psychology2009; 14(4): 447- 53. 30- Bickman L, Rog DJ. Handbook of applied social research methods. London: Sage publication; 1998.

31- Nunnally JC. Psycometric theory.Newyork: Mac grow-hill; 2010.

32- Heesen C, Kasper J, Segal J, Kopke S, Muhlhauser I. Decisional role preferences, risk knowledge and information interests in patients with multiple sclerosis. Mult Scler2004; 10(6): 643-50.

33- Heidari M, Shahbazi. Effect of self-care training program on quality of life of elders. Iran Journal of
Nursing2012; 25(75): 1-8.

34- Darvishpour Kakhki A, Abed Saeedi J, Delavar A, Saeed-O-Zakerin M. Autonomy in the elderly: a phenomenological study. Hakiml2010; 12(4): 1-10.

35- Borg C, Hallberg IR, Blomqvist K. Life satisfaction among older people [65+] with reduced self-care capacity: the relationship to social, health and financial aspects. J Clin Nurs2006; 15(5): 607-18.

36- Ho SC, Woo J, Lau J, et al. Life satisfaction and associated factors in older Hong Kong Chinese. $\mathrm{J} \mathrm{Am}$ Geriatr Soc1995; 43(3): 252-5.

37- Mazloomi SS, Abbacimoghaddam-Niasar A, Saba MAMM, Fallahzadeh H. The relation of knowledge, attitude and self-management behaviors in asthmatic patients with controlling asthma. Zahedan Journal of Research in Medical Sciences2012; 14(1): 49-55.

38- Samadi S, Bayat A, Taheri H, Joneid BS, RoozBahani N. Knowledge, attitude and practice of elderly towards of lifestyle during aging. Journal of Qazvin University of Medical Sciences2007; 11(1). 